\title{
"Jardinería" para la restauración coralina en el Golfo Dulce, Costa Rica: Una prueba práctica
}

\author{
Rodolfo Vargas Ugalde ${ }^{1}$ (D), Cristian Gómez Salas ${ }^{1}$ (D) Carlos Pérez Reyes $^{2-3}$ (D), Erick Umaña Vargas ${ }^{1}$ (iD \\ \& Marco Acosta Nassar ${ }^{1}$ \\ 1. Instituto Nacional de Aprendizaje, Núcleo Náutico Pesquero, Puntarenas, Costa Rica; rvargasugalde@ina.ac.cr, \\ cgomezsalas@ina.ac.cr, umanae7040a@hotmail.com, macostanassar@ina.ac.cr \\ 2. Universidad de Costa Rica, Sede Regional del Pacífico, Núcleo Náutico Pesquero, Puntarenas, Costa Rica; \\ cperezr@uned.ac.cr \\ 3. Universidad Estatal a Distancia, San José Costa Rica; cperezr@uned.ac.cr
}

Recibido 8-VII-2019 • Corregido 16-XI-2019 • Aceptado 12-XII-2019

DOI: https://doi.org/10.22458/urj.v11i3v12i1.2809

\begin{abstract}
Gardening for Coral Reef restoration in the Golfo Dulce, Costa Rica: A practical test". Introduction: The corals reef of Golfo Dulce, South Pacific, Costa Rica, are disappearing due to anthropogenic pollution and sedimentation. Objective: To develop a coral nursery, and establish its viability to subsequently perform a restoration of scleractinian corals in the Pacific of Costa Rica, between December 2014 and May 2016. Methods: We placed the coral nursery structure in the middle of the water column in the Punta Encanto sector, Golfo Dulce using some fragments of coral collected from Porites lobata, Psammocora stellata and Pavona varians, placed in cement "cookies" to make a monthly three-dimensional measurement of growth, and taking water samples for physicochemical analyses. Results: We found that the species Psammocora stellata gave better results in the nursery and that the lowest concentration of nutrients was in the middle of the water column, where silicates were the most abundant throughout the period of the study. In general, we can say that the levels of nutrients are normal in comparison to other studies, however; the phosphate concentration adversely affected all the species in terms of growth, as well as the high salinity. Conclusion: coral gardening is feasable in the Golfo Dulce as a technique for ecological restoration.
\end{abstract}

Keywords: Coral reef, restoration, gardening, phosphate, branching.
RESUMEN. Introducción: Los corales del Golfo Dulce del Pacífico Sur de Costa Rica están desapareciendo debido a la contaminación y sedimentación por origen antrópico. Objetivo: Desarrollar una guardería de corales en Punta Encanto con el fin de establecer su viabilidad y replicarlo en otras partes del Pacífico costarricense, el estudio se realizó entre diciembre del 2014 y mayo del 2016. Metodología: Construimos una estructura de jardinería que colocamos a media columna de agua, utilizamos fragmentos de oportunidad de las especies de coral Porites lobata, Psammocora stellata y Pavona varians que colocamos en galletas de cemento $y$ de esta forma realizamos una medición de su crecimiento en $\mathrm{cm}^{3}$ y tomamos además muestras de agua para su análisis físico químico. Resultados: Determinamos que la especie Psammocora stellata fue la de mostró mejor crecimiento, la menor concentración de nutrientes tuvo lugar a media columna de agua, los silicatos fueron los más abundantes en todo el periodo del estudio. En general, podemos decir que los niveles concentración en nutrientes son normales respecto a los reportados en otros estudios similares. La alta concentración salina y de fosfato afectó negativamente el crecimiento en todas las especies evaluadas. Conclusión: El estudio demostró la factibilidad de realizar jardinerías de coral en el Golfo Dulce como una técnica de restauración ecológica.

Palabras clave: Arrecife de coral, restauración, jardinería, fosfato, ramificado. 
Al menos en 100 países en el mundo, la cobertura de coral vivo es de $284000 \mathrm{~km}^{2}$ del fondo marino, donde Costa Rica cuenta con $970 \mathrm{~km}^{2}$ de estos organismos en sus aguas; ello representa según Asociación Interamericana para la Defensa del Ambiente (AIDA, 2012) \$1 000000 por hectárea de coral en este país, distribuidos en las costas Pacífica y del Caribe. La costa Pacífica de Costa Rica posee $1160 \mathrm{~km}$ de longitud siendo una de las más grandes de América Central. Por su irregularidad marino-costera es común encontrar una alta diversidad de hábitat como arrecifes rocosos, fondos arenosos, zonas rocosas, manglares, fiordos, bosques tropicales y arrecifes coralinos (Cortes, Jiménez, Fonseca, \& Alvarado, 2010).

Los arrecifes coralinos de Golfo Dulce se concentran principalmente en el interior de este como en Mogos, La Viuda, Valinas, Nicuesa, Adela y Sándalo. Se caracteriza por tener de 10 a $15 \mathrm{~km}$ de largo de Noroeste al Sureste, cuya bahía fue de origen tectónico y alcanza profundidades superiores a los 200m en algunas partes (Cortes et al., 2010). Dentro de las especies observadas se tiene a Porites lobata, Pavona gigantea, Pavona varians, Pocillopora damicornis y Psammocora stellata con cobertura viva y muerta.

Cortes et al. (2010) señalan que estos arrecifes comenzaron a crecer hace 5500 años sobre roca basáltica, su mejor crecimiento se dio entre los años 2500 y 500 en el pasado. Posterior a este período los corales comienzan a decrecer, primero por la actividad tectónica, el flujo de nutrientes y sedimentos de los ríos y recientemente por los movimientos de tierra que generan sedimentos, contaminación por PCB, metales pesados y derivados de hidrocarburos. Se suma además la extracción desmedida de organismos como peces, crustáceos, moluscos y corales, el uso de anclas y malas prácticas de manejo provocaron que su cobertura disminuyera considerablemente.

Es una situación que se repite a nivel mundial, debido a la actividad humana se generó el declive de la cobertura viva de estos organismos denominados corales (Guzman, 1991; Jiménez, 2001; Rinkevich, 2005; Shafir, Van Rijn, \& Baruch, 2006; Herler \& Dirnwöber, 2011; Seddon, Griffiths, Soorae, \& Armstrong, 2014; Cummings, Zuke, Stacio, \& Krumholz, 2015; De la Cruz, Rinkevich, Gómez, \& Yap, 2015). AIDA (2012) señala que el problema en Costa Rica es muy grave, se considera que el $93 \%$ de los $970 \mathrm{~km}^{2}$ de los arrecifes coralinos están siendo amenazados por las actividades humanas.

Ante la destrucción de los corales, la restauración es una medida de conservación que no sustituye a la primaria que es la preservación original de los arrecifes, pero procura ayudarle a recuperarse dado que la resiliencia se ha reducido por el impacto antrópico (Guzman, 1991; Young, 2000; Clewel, Aronson, \& Winterhalder, 2004; Shafir et al., 2006; De la Cruz et al., 2015). Existen varias técnicas de restauración para las poblaciones de corales, pero la más exitosa según Becking (2015) es la "Jardinería de Corales" hasta el momento, dado que la técnica ofrece una alta tasa de supervivencia y estos resultados lo confirma Rinkevich (2000) en su trabajo. Dentro de los razonamientos para llegar a dicha conclusión están: (1) ayudan al contacto con nutrientes en el agua, (2) evita la acción coralívora y (3) recupera fragmentos de coral vivo desprendidos que estarían condenados a morir. Nedimyer, Gaines y Roach (2011) la llaman la maricultura de los corales donde los fragmentos asexuales fueron originados por la bioerosión, rupturas durante las tormentas y la acción de peces coralívoros (Guzmán, 1991).

Los corales brindan bienes y servicios como (1) protección costera por huracanes y tormentas tropicales, (2) fuente abundante de pesca y valiosa información para la investigación médica, (3) turismo, recreación y esparcimiento (Levi, Shaish, Haim, \& Rinkevich, 2010) y (4) mantenimiento de otros hábitats ricos en diversidad biológica. Por las razones anteriores, los arrecifes coralinos los llaman los bosques lluviosos tropicales del mar (Guzmán, 1991; Shafir et al., 2006; Baums, 2008; De la Cruz et al., 2015).

Bajo esa misma línea, este trabajo pretende determinar la viabilidad de implementar la técnica de jardinería de corales en el Golfo Dulce del Pacífico Sur de Costa Rica con el fin de ser 
replicado en otras zonas costeras del país con el objeto de repoblar arrecifes coralinos en zonas impactadas, considerando además que no existe ningún esfuerzo de jardinería de corales en el lado del Océano Pacífico en el continente americano (J. Alvarado, comunicación personal, 18 de abril, 2018), por lo que es necesario realizar este trabajo.

\section{MATERIALES Y MÉTODOS}

Selección del sitio: La investigación se realizó en Punta Encanto de Golfo Dulce en las coordenadas $08^{\circ} 38^{\prime} 54.5^{\prime \prime} \mathrm{N} \& 83^{\circ} 15^{\prime} 07.5^{\prime \prime} \mathrm{W}$, una zona rodeada de arrecifes rocosos y cerca de la costa a una profundidad máxima de $12 \mathrm{~m}$ en marea baja. El proyecto inició el 5 de diciembre 2014 y finalizó el 5 de mayo 2016 cuando los corales empezaron a disminuir su crecimiento hasta morir.

Especies: Se localizó varias especies de coral mediante buceo exploratorio en visitas realizadas a zonas como Adela, Mogos, Esquinas, Sándalo, Valinas y Nicuesa en el Golfo Dulce, se utilizó parámetros de densidad promedio y frecuencia de avistamiento, para seleccionar las especies que mejor podrían responder al cultivo y se conviertan en donantes. Mediante la observación directa se caracterizó los principales organismos coralívoros e invasores de estas especies coralinas. Los sitios de mayor cantidad de fragmentos de oportunidad fueron Sándalo y Punta Adela, donde se seleccionaron las especies Porites lobata, Psammocora stellata y Pavona varians por presentar mayor resistencia y abundancia, y por la recomendación de los investigadores del Centro de Investigación en Ciencias del Mar y Limnología (CIMAR) de la Universidad de Costa Rica.

Construcción e instalación de la guardería: Se construyó un marco en tubería PVC de $150 \mathrm{~cm} \times 150 \mathrm{~cm}$ conectado por una red de plástico de un diámetro de $1 \mathrm{~cm}$ que fue adherida al marco con gazas plásticas de $12,7 \mathrm{~cm}$ de largo. En cada extremo, se le amarró $150 \mathrm{~cm}$ de cabo marino sansón de $1,9 \mathrm{~cm}$ de diámetro con el fin de amarrar el ancla en la parte inferior y las boyas en la parte superior de la cuadrícula. Para su instalación en el agua, la cuadrícula quedó suspendida por cuatro envases llenos de aire en cada extremo del marco a $5 \mathrm{~m}$ de la superficie y sostenida por un ancla tipo tornillo en fondo arenoso con un cabo de $5 \mathrm{~m}$ de que se une a cuatro cabos que sostienen la estructura flotante (Azueta, 2009; Edwards, 2010; Levi et al., 2010). La profundidad promedio en marea alta fue de $12 \mathrm{~m}$ y la estructura estuvo siempre suspendida a $5-6 \mathrm{~m}$ en la columna de agua por medio de cuatro boyas en los extremos del cuadrante de PVC. Se marcó la posición con una boya en la superficie para su localización visual además de la respectiva georreferenciación (Fig. 1).

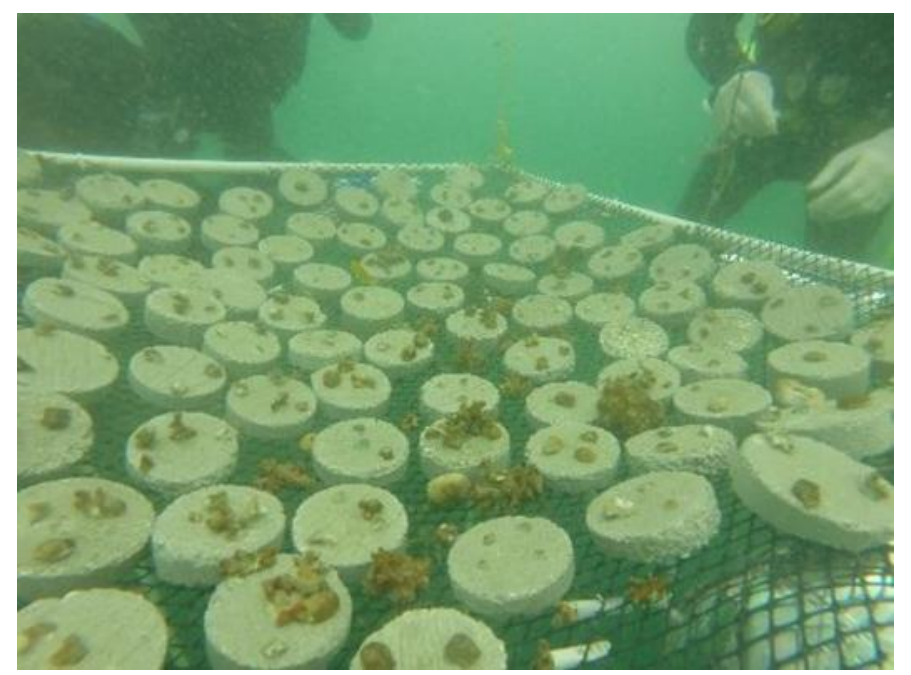

Fig. 1. Estructura flotante donde se realizó el cultivo de corales. 
Por otra parte, las estructuras para fijación de corales "galletas" se construyeron se realizaron empleando moldes de un tubo de PVC de $10 \mathrm{~cm}$ cuyo corte era de $2,54 \mathrm{~cm}$ de alto. Estos moldes se rellenaron con una mezcla de cemento, arena y piedra quinta. Posteriormente se insertó sobre esta preparación fresca, un espander de $2.5 \mathrm{~cm}$ de largo. A las galletas se les fijó un fragmento de coral vivo, de 3 a $5 \mathrm{~cm}$ en promedio, para su fijación se aplicó goma de pegado rápido, según lo propuesto por Edwards y Gómez (2007) y Levi et al. (2010) a 70 fragmentos de coral distribuidos en 22 de la especie Pavona varians y 28 de la especie Porites lobata. Mientras que la especie Psammocora stellata se cosechó mediante una cuerda de $0,5 \mathrm{~cm}$, donde se amarraron 20 fragmentos de $5 \mathrm{~cm}$ de forma colgante en la parte superior de la estructura, esto último siguiendo el método de Levi et al. (2010). Todas las galletas fueron marcadas y medidas para darles el debido seguimiento en días posteriores.

Tasa de crecimiento: Se procedió a realizar una visita por mes desde el 2015 al 2016, para un total de 17 visitas donde se removieron algas e invasores por medio de cepillos y espátulas, estos epibiontes crecían sobre las galletas y afectaban el crecimiento de los corales. Además, en seis de las 17 visitas se tomaron datos como el volumen de crecimiento de cada especie $\mathrm{en}^{3}$ (midiendo el largo, alto y ancho), con la ayuda de un vernier, un único investigador realizó todas las lecturas en este período. La idea fue comparar las colonias en forma tridimensional como lo señala Edmund y Gates (2002) para determinar cuál especie creció de mejor forma y dar por válida la técnica de cultivo en la zona.

Monitoreo: Durante la medición del crecimiento se registraron parámetros físicos químicos del agua, tales como la temperatura en grados Celsius, salinidad en ppm, pH y conductividad por medio de multiparámetro portátil Hanna DiST ${ }^{\circledR}$ tester DiST 1 y 4 , se agrega la biodiversidad asociada a la estructura y penetración de luz por medio del disco Sechii, lo anterior con el fin de relacionar alguno de estos factores con el crecimiento de las especies de coral. Además, se tomaron muestras de agua de mar en botellas oscuras de $1 \mathrm{~L}$ a 3 estratos de la columna de agua: superficie, media y fondo. Para determinar los niveles de Fosfatos $(\mu \mathrm{mol} / \mathrm{L})$, Silicatos $(\mu \mathrm{mol} / \mathrm{L})$, Amonio $(\mu \mathrm{mol} / \mathrm{L})$, Nitritos ( $\mu \mathrm{mol} / \mathrm{L}$ ), Nitratos ( $\mu \mathrm{mol} / \mathrm{L}$ ) por medio de un Autoanalizador de Flujo Constante (FIA por sus siglas en inglés) Modelo QuikChem 8500 Series 2 de la marca Lachat Instruments en el Centro de Investigación en Ciencias del Mar y Limnología (CIMAR) de la Universidad de Costa Rica. Esto permitió establecer si la presencia o ausencia de alguna de estas variables afectaría el crecimiento de los corales en la guardería.

Correlaciones: Una vez tabulado los datos se procedió a realizar pruebas de correlación de Spearman $(r)$ para determinar la relación entre las variables físico químicas obtenidas con respecto al crecimiento mostrado por los corales en la jardinería. Además, se realizó la prueba de Chi cuadrado para comparar los estándares de calidad de agua versus los resultados obtenidos, todo lo anterior gracias al programa estadístico Instat+ v3.33 (Statistic).

Ética, conflicto de intereses y declaración de financiamiento: Los autores declaran haber cumplido con todos los requisitos éticos y legales pertinentes, tanto durante el estudio como en el manuscrito; que no hay conflictos de interés de ningún tipo, y que todas las fuentes financieras se detallan plena y claramente en la sección de agradecimientos. Asimismo, están de acuerdo con la versión editada final del documento. El respectivo documento legal firmado se encuentra en los archivos de la revista. 


\section{RESULTADOS}

Tasa de crecimiento: En este trabajo la tasa de crecimiento fue mayor en la especie Psammocora stellata $84,899 \pm 36,7 \mathrm{~cm}^{3} / a n ̃ o ~(n=20)$ (promedio \pm desviación estándar); mientras que la especie Pavona varians mostró menor tasa de crecimiento $5,158 \pm 1,95 \mathrm{~cm}^{3} / a n ̃ o ~(n=22)$ (Fig. 2). Las especies Pavona varians y Porites lobata tuvieron un crecimiento sostenido desde diciembre 2014 hasta agosto 2015, posterior a este mes comenzó a decaer hasta llegar a cero coberturas vivas. Este fenómeno concuerda con una importante marea roja que se presentó en ese mismo mes de agosto. Caso contrario, la especie Psammocora stellata donde el crecimiento se mantuvo considerablemente hasta el final.

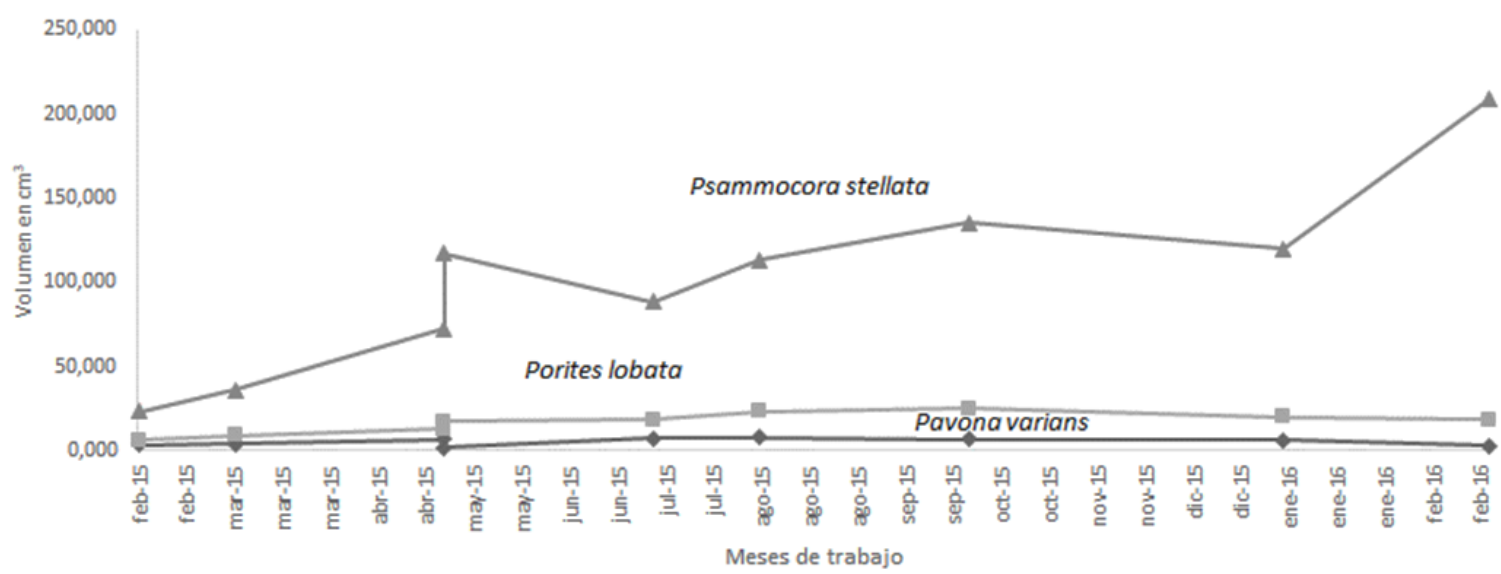

Fig.2. Crecimiento volumétrico $\left(\mathrm{cm}^{3}\right)$ de las tres especies de coral cultivadas en el Golfo Dulce de Puntarenas. 2015-2016.

Monitoreo: Al unificar todos los parámetros según nivel en la columna de agua, se determinó que la mayor concentración de nutrientes se dio en la superficie $(2,285 \pm 1,564)$, seguido del fondo $(1,978 \pm 1,348)$ y finalmente la de menor registro a media columna de agua $(1,460 \pm 0,5479)$ y se mantuvo constante en todo el período. Es precisamente en este último estrato donde se colocó la estructura. El comportamiento de los nutrientes durante las seis lecturas realizadas revela que agosto es donde se obtiene el máximo a nivel de fondo. Mientras que en diciembre donde se registra la mayor cantidad de nutrientes en la superficie y octubre la menor lectura en la parte media de la columna de agua (Fig. 3).

Analizando cada nutriente, se determinó que el elemento de mayor concentración en todo el periodo correspondió a los silicatos $(9,636 \pm 3,021)$ en octubre, mientras que en el segundo lugar correspondió al amonio $(5,556 \pm 0,0818)$ en mayo y los nitratos en tercer lugar en octubre $(1,555 \pm 0,6400)$ (Fig. 4).

En términos generales existen diferencias significativas entre los estándares normales y la estacionalidad (Prueba Chi; $p=2,393 \mathrm{E}-17$ ) pero no incumplen con valores registrados en otros trabajos. Se hizo una revisión al respecto y se determinó que los valores obtenidos de nutrientes se encuentran en un ámbito aceptable y aunque en el caso de los compuestos nitrogenados existe preocupación, los valores realmente son bajos con respecto a otros países (Cuadro 1). 


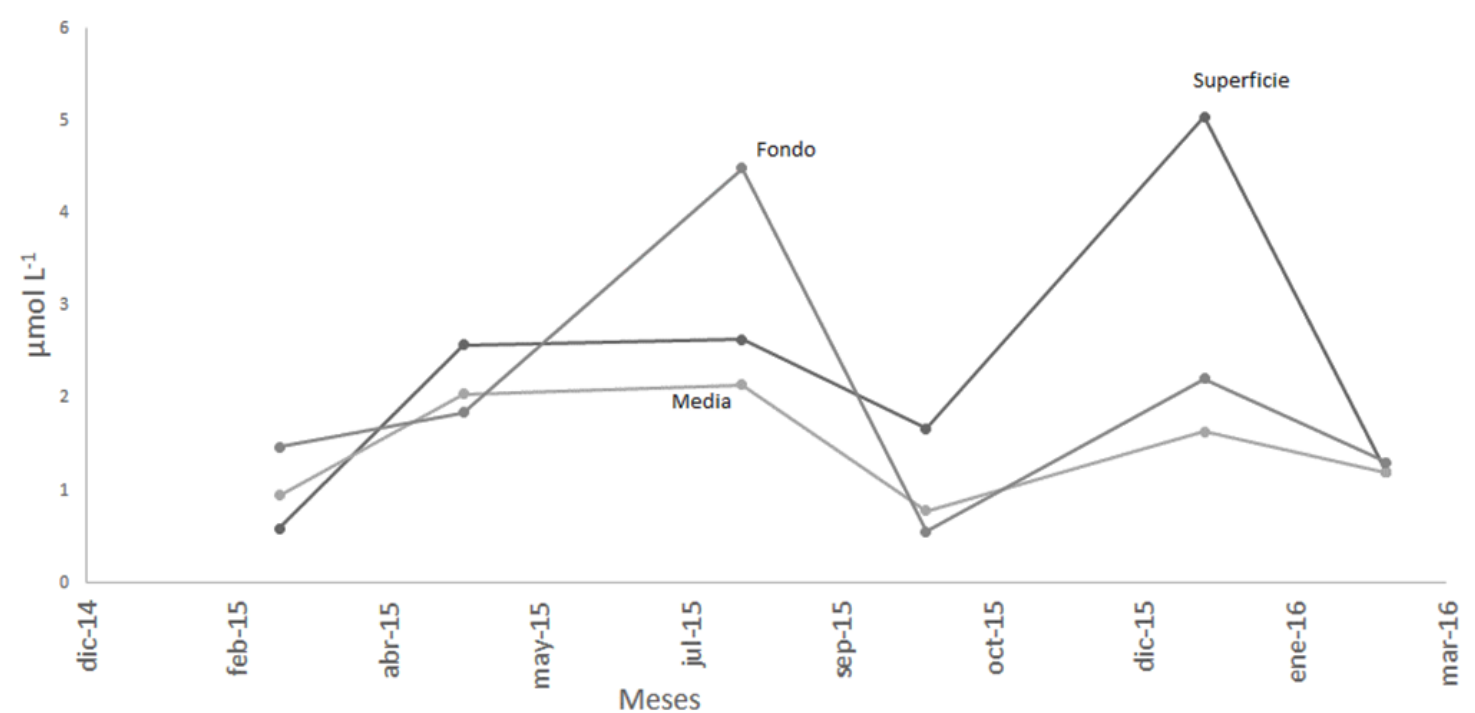

Fig. 3. Concentración de nutrientes en la columna de agua en Punta Encanto de Golfo Dulce en el período 2015-2016.

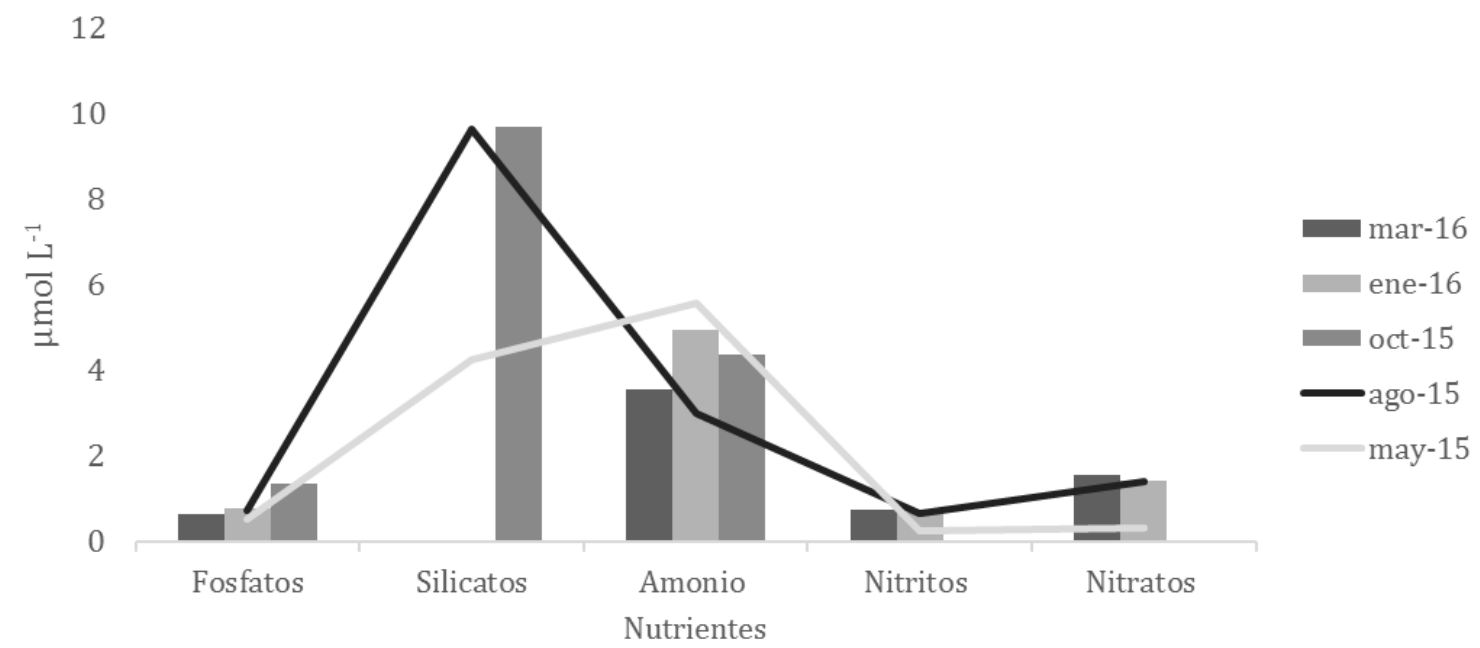

Fig. 4. Tipos de nutrientes según concentración por meses de lectura en Punta Encanto de Golfo Dulce. Año 2015-2016.

La tendencia de los parámetros físicos del agua es distinta entre sí. Por ejemplo, en la concentración de oxígeno es importante en marzo, la salinidad aumenta en febrero, pero comienza a disminuir en los meses de julio y octubre respectivamente. La lectura más importante de temperatura se presenta en enero y marzo, pero baja en mayo considerablemente. El pH no sufre variaciones significativas en todo el año, manteniéndose siempre en niveles superiores a ocho. Situación distinta se da con el disco Secchi y la penetrancia de la luz, donde el valor más alto se da en marzo y el menor se da en mayo cuando se registraron las primeras lluvias y marea roja.

Los parámetros físicos medidos en el Punta Encanto, al ser comparados con los estándares nacionales e internaciones indican que las condiciones del agua son normales y no hay variaciones que Ileguen a causar un estrés para los corales (Prueba Chi; $p=1,292 \mathrm{E}-08$ ) (Martínez, Alvarado, \& Senior, 2001; Coll, Cortes, \& Sauma, 2004; Acuña et al., 2008; Arévalo et al., 2015) (Cuadro 2). 


\section{CUADRO 1}

Parámetros de los nutrientes en general en Punta Encanto de Golfo Dulce en el período 2015-2016

\begin{tabular}{lcl}
\hline Parámetros & Intervalo $(\boldsymbol{\mu m})$ & \multicolumn{1}{c}{ Cita } \\
\hline Amonio & $0,1-10$ & Jompa \& McCook, 2002 \\
& $0,53-7,69$ & Torres \& Calva, 2012 \\
$0-0,305$ & Alvarado, 2006 \\
$0,03-0,10$ & Hernández et al., 2011 \\
& $0,08-8,11$ & Este estudio \\
\hline Nitritos & $0,03-0,15$ & Voss \& Richardson, 2006 \\
& $0,05-0,32$ & Torres \& Calva, 2012 \\
& $0-3,360$ & Alvarado, 2006 \\
& $0,08-0,785$ & Este estudio \\
\hline Nitratos & 0,4 & Schaffelke et al., 2003 \\
& $0,28-1,36$ & Voss \& Richardson, 2006 \\
& $0,05-2,43$ & Torres \& Calva, 2012 \\
$0-0,363$ & Alvarado, 2006 \\
& $0,122-1,82$ & Este estudio \\
\hline Ortofosfatos & $0,08-0,12$ & Jompa \& McCook, 2002 \\
& $0,03-0,1$ & Furnas et al., 1996 \\
& $0,41-2,92$ & Flores et al., 2001 \\
& $0,033-0,453$ & Alvarado, 2006 \\
$0,068-2,93$ & Este estudio \\
\hline Silicato & $10,9-18,1$ & Álvarez \& Chee, 1976 \\
& $0-5,67$ & Acuña et al., 2008 \\
& $2,098-35,360$ & Alvarado, 2006 \\
$0-21,26$ & Este estudio \\
\hline
\end{tabular}

Invasores: Se detectó presencia de invasores tales como: gusanos tubulares, cirripedos, percebes, macroalgas, nudibranquios, cangrejos pequeños, caballitos de mar y esponjas principalmente sobre las galletas de cemento. Su aparición comenzó a detectarse en el primer mes de colocadas las galletas. De todos ellos, los gusanos tubulares, esponjas, cirripedos, percebes y algas fueron removidos con el fin de reducir el daño del coral y la presión depredatoria. En ese mismo primer mes se comenzó a observar crecimiento en las colonias de coral en el cultivo.

Correlación: Dentro de las relaciones entre el crecimiento de cada especie con respecto a los parámetros físicos y químicos del agua, existe una notable influencia del amonio, nitratos y nitritos sobre el crecimiento de las especies de coral, donde al incrementarse su concentración favoreció el crecimiento de las especies de Porites lobata (Spearman, $\mathrm{r}=0,7$ ) y Psammocora stellata (Spearman, $r=0,8)$, caso contrario la especie Pavona varians disminuyó (Spearman, $r=-0,2)$. Mientras que el $\mathrm{pH}$ y la Temperatura al aumentar sus valores ayuda el crecimiento de las especies, pero principalmente a la especie Pavona varians ( $r>0)$.

Al incrementarse la salinidad, todas las especies disminuyeron la tasa de crecimiento, especialmente Psammocora stellata (Spearman, $\mathrm{r}=-0,8$ ), el oxígeno disuelto a niveles de sobresaturación $(8,36 \mathrm{mg} / \mathrm{L})$ afectó el crecimiento de todas las especies, en particular a la especie Porites lobata (Spearman, $r=-0,6$ ). El fosfato afectó el crecimiento de las tres especies de coral. En Pavona varians se observó que a menor penetrancia de la luz se presentó el mayor crecimiento, Porites lobata y Psammocora stellata, ante una mayor claridad experimentaron mayor crecimiento se registra (Cuadro 3). 


\section{CUADRO 2}

Comportamiento de los parámetros físicos del agua durante seis lecturas en Punta Encanto de Golfo Dulce, año 20152016

\begin{tabular}{lcccccc}
\hline \multicolumn{1}{c}{ Día } & $\begin{array}{c}\text { Oxígeno } \\
(\mathbf{m g} / \mathbf{l})\end{array}$ & $\begin{array}{c}\text { Temperatura } \\
\left({ }^{\circ} \mathrm{C}\right)\end{array}$ & $\mathbf{p H}$ & Secchi $(\mathbf{m})$ & $\begin{array}{c}\text { Salinidad } \\
(\mathbf{p p m})\end{array}$ & $\begin{array}{c}\text { Conductividad } \\
(\boldsymbol{\mu s} / \mathbf{c m})\end{array}$ \\
\hline febrero- 2015 & 8,22 & 30,6 & 8,4 & 9 & 31 & 2000 \\
marzo-2015 & 9,78 & 30,1 & 8,6 & 8 & 31 & 2000 \\
mayo-2015 & 9,44 & 30,7 & 8,54 & 4 & 31 & 2666 \\
julio-2015 & 9,32 & 30,9 & 8,5 & 7 & 28 & 1999 \\
agosto-2015 & 4,43 & 31,4 & 8,52 & 5 & 30 & 2000 \\
octubre-2015 & 5,00 & 31,0 & 8,66 & 10 & 30 & 2000 \\
enero-2016 & 4,40 & 31,6 & 8,93 & 12 & 30 & 4000 \\
marzo-2016 & 5,82 & 30,3 & 8 & 5,5 & 30 & 1890 \\
mayo 2016 & 5,80 & 30,1 & 8 & 7,722 & 29,556 & 2272,778 \\
Promedio & 6,91 & 30,7 & 8,461 & 1 & 35 & $2000-3000$ \\
Agua de mar & 6,39 & 30 & 8,4 & & & 25 \\
\hline
\end{tabular}

\section{CUADRO 3}

Parámetros físicos correlacionados al crecimiento de las distintas especies de coral cultivadas en Punta Encanto, Puntarenas, 2016

\begin{tabular}{lccc}
\hline Parámetros & Pavona varians & Porites lobata & Psammocora stellata \\
\hline Oxigeno & $-0,1622$ & $-0,6551$ & $-0,1214$ \\
pH & 0,7712 & $-0,0591$ & 0,2988 \\
Salinidad \% & $-0,3073$ & $-0,3750$ & $-0,8355$ \\
Temperatura & 0,8646 & 0,0883 & 0,2244 \\
Sechii (m) & $-0,1218$ & 0,1221 & 0,3638 \\
Fosfatos & $-0,3835$ & $-0,3835$ & $-0,3835$ \\
Silicatos & 0,1809 & 0,1503 & 0,0055 \\
Amonio & $-0,1536$ & 0,7969 & 0,6973 \\
Nitritos & $-0,3053$ & 0,8270 & 0,8758 \\
Nitratos & $-0,2880$ & 0,7690 & 0,8042 \\
\hline
\end{tabular}

\section{DISCUSIÓN}

Tasa de crecimiento: El crecimiento responde a variables ambientales como la concentración de nutrientes, salinidad, luz y oxígeno (Edmunds \& Gates, 2002; Herler \& Dirnwöber, 2011). Es en el cuarto mes donde el crecimiento se detiene y comienza a morir la colonia de forma paulatina. En el trabajo de Becking (2015) se observó este patrón, pero a las siete semanas, momento en que el crecimiento se detuvo por completo. En este trabajo solo en la especie Psammocora stellata se obtuvo un buen crecimiento y este hallazgo lo respalda Rinkevitch (2008) quien señala que los corales ramificados son especies claves en las técnicas de restauración dado que crecen más rápido y en especial en las puntas.

Otro factor importante fue las variaciones en la penetración de la luz, la especie Pavona gigantea crece mejor cuando la penetración de luz es menor, mientras que las especies Psammocora stellata y Porites lobata crecieron mejor cuando la penetración de luz fue mayor, dado que estas son más de aguas someras, mientras que Pavona varians es de mayor profundidad. En el caso de las especies ramificadas, Guzman (1991) y Baums (2008) mencionan que estas especies 
demuestran poco potencial reproductivo sexual y su estrategia es más bien la vía asexual por fragmentación dado que posee una alta resistencia a la destrucción de coralívoros y especies herbívoras, por tanto, el valor adaptativo de estas especies es más alto con respecto a otras. Bassey Fallas (2010) señala que Psammoroca stellata es la octava especie de mayor abundancia en algunas partes del Pacífico (36\%), lo que la convierte en una candidata de repoblamiento urgente, por encima inclusive de Pocillopora damicornis. Se recomienda hacerlo en los meses de mayor intensidad de luz, pero al momento del trasplante que sea en meses de menor temperatura como sucede en la época lluviosa, ya que esto reduce la mortalidad (Edwards, 2010).

Monitoreo: En este trabajo se observó en términos generales que la presencia de compuestos nitrogenados estimuló el crecimiento de las especies Porites lobata y Psammocora stellata, resultado similar al reportado por parecido al de Shafir et al. (2006) con tres especies de coral en Caribe. Los nutrientes también son un problema ya que promueven el desarrollo de macroalgas quienes cubren al coral e impiden que las zooxantelas realicen fotosíntesis y por ende la disminución en el crecimiento de los corales en la jardinería. Esto se traduce en altos costos y un mayor tiempo de cultivo, lo cual daría oportunidad a depredadores e invasores (Shafir et al., 2006; Edwards, 2010).

El amonio es compuesto altamente perjudicial en el desarrollo de la vida en el mar por su toxicidad, fue en los meses de enero, octubre y mayo las lecturas superaron los $5 \mu \mathrm{mol} / \mathrm{L}$, en el fondo y la superficie, pero no donde estaba la estructura que albergaba los corales cosechados a media columna de agua. En el caso de los silicatos solo se registró un valor de $21 \mu \mathrm{mol} / \mathrm{L}$ en agosto a nivel de superficie lo cual es indicativo de una alta productividad. Finalmente, los fosfatos alcanzaron valores entre $1,5 \mu \mathrm{mol} / \mathrm{L}$ y $2,9 \mu \mathrm{mol} / \mathrm{L}$ sin causar eutrofización ya que se requirieren concentraciones $6 \mu \mathrm{mol} / \mathrm{L}$ para que ocurra este proceso. En términos generales, el mes de octubre fue el momento donde el crecimiento comenzó a detenerse debido al estrés por nutrientes.

En el caso de los nitratos, valores superiores a $0,1 \mu \mathrm{mol} / \mathrm{L}$ revelan una alta productividad para el fitoplancton y en este trabajo los valores registrados en Punta Encanto superan por mucho esta cifra. En el caso de los fosfatos, las concentraciones de $0,5 \mu \mathrm{mol} / \mathrm{L}$ se considera baja productividad debida a que en promedio el valor registrado fue de $0,142 \mu \mathrm{mol} / \mathrm{L}$. La concentración promedio de los Silicatos fue de $1,889 \mu \mathrm{mol} / \mathrm{L}$, se establece que valores menores a $15 \mu \mathrm{mol} / \mathrm{L}$ es baja (Martínez et al., 2001; Coll et al., 2004; Acuña et al., 2008; Arévalo, Maldonado, Iglesias, Cabrera, \& Concepción, 2016). Los nutrientes muestran valores preocupantes en las concentraciones de Nitratos y Nitritos, la presencia de fosfatos fue relevante en este trabajo (Cuadro 1). Según Alvarado (2006) los fosfatos tienen un efecto inhibidor de crecimiento en los corales, a pesar de que las lecturas no generaron valores cercanos al límite permitido, si afectaron el desarrollo de estos.

Correlación: Todos los parámetros fisicoquímicos analizados en este trabajo afectaron el crecimiento de cada especie, aunque demandan mayor atención aquellos que interfieren en el crecimiento como los fosfatos, nitratos y nitritos. La acidez, según AIDA (2012), detiene el crecimiento de los corales y hacen referencia al caso de la Gran Barrera Australiana donde el aumento de la acidez ha desacelerado en un $20 \%$ el crecimiento de los corales en los últimos 16 años. Rinkevich (2005) menciona que los parámetros fisicoquímicos como los analizados en este trabajo, pueden ser importante entre un lugar y otro, por eso en todo proyecto de Jardinería debe ser medidos de manera constante.

La temperatura tuvo un efecto favorable en el crecimiento de los corales cultivados, a pesar de estar cerca del valor crítico que son los $30^{\circ} \mathrm{C}\left(86^{\circ} \mathrm{F}\right)$. Se debe tener presente que la relación del endosimbionte entre las especies de coral es crucial para que estos se mantengan vivos a temperaturas menores de los $30^{\circ} \mathrm{C}$, ya que pueden no solo producir el esqueleto de carbonato de calcio sino también la fotosíntesis, existe, por tanto, una selección específica del simbionte por parte de cada especie de coral. Eso explica el por qué la especie Pavona varians tuvo una mejor reacción 
al incrementarse de la temperatura del agua, con respecto a las otras dos. La alta temperatura puede provocar distintas reacciones sobre el simbionte según Baums (2008): (1) la resistencia de algunas cepas de zooxantelas, (2) los mismos corales protegen más a sus zooxantelas, (3) los corales se asocian con nuevas cepas de zooxantelas y (4) las colonias de coral se vuelven más resistentes a las altas temperaturas por sí mismos. Lo anterior podría ser interpretado como una evidencia de un efecto fundador de especies más resistentes a las altas temperaturas por deriva génica, en momentos en que el mar aumenta considerablemente su temperatura por el cambio climático. Esa variabilidad genética puede ser impulsada por las jardinerías de coral ya se pueden implantar ejemplares de coral de distintos lugares en áreas que requieren ser repobladas, evitando de paso la endogamia, puesto que en todo momento se pueden seleccionar donantes bajo criterios científicos.

Otras consideraciones no menos importantes las menciona Young (2000) y Rinkevich (S.A.) al indicar que la Jardinería de corales al realizar el trasplante, se salta la etapa de la sucesión natural y la repoblación se da en un tiempo mucho menor. Claro está siempre existen problemas como la acción de especies coralívoras que tienen un efecto adverso en el crecimiento de los corales trasplantados post cultivo.

La Jardinería de corales por tanto es factible de realizarla en el Golfo Dulce en Costa Rica, no solo por su bajo costo de elaboración y sino también por el crecimiento observado durante esta investigación y las condiciones físico química que ofrece el agua de la zona, esto último es un factor fundamental según Nedimyer et al. (2011). Comparado con otros procesos de restauración ecológica, como siembra de manglar y pasto marino, la jardinería de corales resulta más económica (Edwards \& Gómez, 2007).

Este tipo de esfuerzos deben ser bien encaminados bajo el amparo de políticas ambientales, por ello Costa Rica presentó ante la Organización Mundial de las Naciones Unidas (ONU) un Plan Integral del Objetivo 14 de la Agenda 2030 (MIDEPLAN, 2018) donde se incluyó este proyecto de Jardinería de Corales en el Golfo Dulce por parte del Instituto Nacional de Aprendizaje como un compromiso ante la comunidad científica mundial. Cortes et al. (2010) indican la urgencia de este tipo de acciones y solicitan la inclusión de Golfo Dulce y la Isla del Coco en un plan de conservación y manejo.

Con este tipo de proyectos se contribuye a brindar espacios para que los organismos marinos realicen sus ciclos biológicos incrementando por ende la diversidad biológica del sitio restaurado (Edwards \& Gómez, 2007). En Costa Rica se tienen lugares idóneos para ello, se menciona el Parque de Cahuita en la provincia de Limón o el tómbolo de la Ballena en el Parque Marino Ballena de la Provincia de Puntarenas. Finalmente se recomienda continuar con la prueba de distintas estructuras como plataformas, block de fondo, antenas de televisión y tendederos con varias especies de coral para establecer cual es más adecuada para realizar los cultivos, con ello trasladar este esfuerzo a otras zonas de Costa Rica.

\section{AGRADECIMIENTOS}

Este trabajo fue posible gracias a la coordinación de Marjorie Jiménez Castro y Álvaro Morales Ramírez para poner en marcha la investigación, impulsada por la iniciativa de Rodolfo Vargas Ugalde y de sus estudiantes Emanuel Castro Alvarado y Licie Méndez Benavidez. Así como la asesoría técnica y científica de Nufar Charuvi $(\mathrm{QdDg})$, Juan José Alvarado Barrientos y Jorge Cortes Núñez. A Juan Guillermo Sagot por su ayuda en el análisis de calidad de aguas en el CIMAR. Mencionamos también al Hotel Nicuesa y Don Abraham por brindarnos un valioso apoyo en cuanto a la embarcación y un lugar estratégico para el trabajo de campo. En la persona de Guido Saborío Rodríguez y Geiner Barquero Vanegas del Área de Conservación de Osa (ACOSA) del MINAE, no solo por los permisos de investigación sino por su visión y compromiso con la propuesta. A las distintas 
personas que nos colaboraron en el diseño y registro de los datos, entre las que citamos a María Vargas Obando, Hannia Ferreto Mora, Loriana Campos Sánchez, Stefany Hernández Díaz, Mónica Fuentes, Noelia González Picado, Sharon Alfaro, Keyla Jiménez Vindas, Gabriela Acosta, Fabiola Arce Núñez y Miguel Navarro Salas.

\section{REFERENCIAS}

Acuña, J., García, J., Gómez, E., Vargas, J., \& Cortés, J. (2008). Parámetros físico químicos en aguas costeras de la Isla del Coco, Costa Rica (2001-2007). Revista de Biología Tropical, 52(Suppl 2), 49-56.

Alvarado, J. J. (2006). Factores físico - químicos y biológicos que median en el desarrollo de los arrecifes y comunidades coralinas del Parque Nacional Marino Ballena, Pacífico Sur, Costa Rica (Tesis de Maestría). Universidad de Costa Rica, San José, Costa Rica.

Álvarez, S., \& Chee, A. (1976). Distribución superficial de fosfatos y silicatos en Bahía San Quintín. Ciencias Marinas, 3(1), 51-61. DOI: $10.7773 / \mathrm{cm} . v 3 i 1.293$

Arévalo, W., Maldonado, M., Iglesias, S., Cabrera, C., \& Concepción, L. (2016). Evaluación de la calidad ambiental del ecosistema de la bahía de Ancón durante octubre 2013. Revista del Instituto de Investigación de la Facultad de Ingeniería Geológica, Minera, Metalúrgica y Geográfica, 18(36).

Asociación Interamericana para la Defensa del Ambiente (AIDA). (2012). Los arrecifes de coral en Costa Rica, valor económico, amenazas y compromisos legales internacional que obligan a protegerlos. San José, Costa Rica: Conservation International.

Azueta, J. (2009). Coral reef replenishment manual. Belize City, Belize: OSPESCA National Coordinator.

Bassey Fallas, G. (2010). Evaluación ecológica de los arrecifes y comunidades coralinas de las Islas Murciélago y Sección norte de la Península de Santa Elena, Pacífico de Costa Rica (Tesis de Maestría). Universidad Nacional de Costa Rica, Puntarenas, Costa Rica.

Baums, I. (2008). A restoration genetics guide of coral reef conservation. Molecular Ecology, 17, 2796-2811. DOI: 10.1111/j.1365-294X.2008.03787.x

Becking, L. (2015). Coral restoration Bonaire. An evaluation of growth, regeneration and survival. Rapport C152/15, 32.

Clewel, A., Aronson, J., \& Winterhalder, K. (2004). Principios del SER international sobre la restuaración ecológica. Carolina, EEUU: Sociedad internacional para la restauración ecológica.

Coll, M., Cortés, J., \& Sauma, D. (2004). Características físico - químicas y determinación de plaguicidas en el agua de la laguna de Gandoca, Limón. Revista de BiologíaTropical, 52(Supl 2), 33-42.

Cortes, J., Jiménez, C., Fonseca, A., \& Alvarado, J. (2010). Status and conservation of coral reefs in Costa Rica. Revista de BiologíaTropical, 58(1), 33-50. DOI: 10.15517/rbt.v58i1.20022

Cummings, K., Zuke, A., Stacio, B., \& Krumholz, J. (2015). Coral Growth assessment on an establesihed artificial reef in Antigua. Ecological Restoration, 33(1), 90-95. DOI: 10.3368/er.33.1.90

De la Cruz, D., Rinkevich, B., Gómez, E., \& Yap, H. (2015). Assesing an abridged nursery phase for slow growing corals used in coral restoration. Ecological Engineering, 84, 408-415. DOI: 10.1016/j.ecoleng.2015.09.042

Edmunds, P., \& Gates, R. (2002). Normalizing physiological data for scletactinian corals. Coral Reefs, 21, 193-197.

Edwards, A. (2010). Reef rehabilitation Manial. Sta Lucia, Australia: Coral Reef Targeted \& Capacity Building for Management Program (GEF). 
Edwards, A., \& Gómez, E. (2007). Restauración Arrecifal conceptos y recomendaciones: tomando decisiones de gestión sensatas ante la incertidumbre. Sta Lucía, Australia: Coral Reef Targeted Research \& Capacity Building for Management Program.

Flores, G., Carrasco, N., \& Sarmiento, M. (2001). Oxígeno disuelto, nutrientes y clorofila A del Mar Peruano en Mayo 2000. Informe Instituto del Mar del Perú, 163, 25-34.

Furnas, M., Mitchell, A., \& Skuza, M. (1996). Shelf scale nitrogen and phosphorus budgets for the central Great Barrier Reef (16-195). In H. Lessions \& I. Macintyre (Eds.), Proceedings of the 8th International Coral Reef Symposium, Panama (pp. 809-815). Balboa, Panamá: Smithsonia Tropical Research Institute.

Guzman, H. (1991). Restoration of Coral Reefs in Pacifico Costa Rica. Conservation Biology, 5(2), 189-195. DOI: 10.1111/j.1523-1739.1991.tb00123.x

Herler, J., \& Dirnwöber, M. (2011). A simple technique for measuring bouyant weight increment of entire, trasplanted coral colonies in the field. Journal of Experimental Marine Biology and Ecology, 407, 250-255. DOI: 10.1016/j.jembe.2011.06.022

Hernández, G., Lango, F., Castañeda, M., Zamora, J., \& Maldonado, J. (2011). Cultivo del coral cuerno de alce Acropora palmata en un sistema recirculaedo utilizando agua de mar sintética. Revista de Biologia Marina y Oceanografía, 46(3), 477-482. DOI: 10.4067/S0718-19572011000300017

Jiménez, C. (2001). Arrecifes y ambientes coralinos de Bahía Culebra. Pacífico de Costa Rica; aspectos biológicos, económico-recreativo y de manejo. Revista de BiologíaTropical, 49(2), 215-231.

Jompa, J., \& McCook, L. (2002). The effects of nutrients and hervibory on competition between a hard coral (Porites cylindrica) and brown alga (Lobophora variegata). Limnology and Oceanography, 46, 527-534. DOI: 10.4319/lo.2002.47.2.0527

Levi, G., Shaish, L., Haim, A., \& Rinkevich, B. (2010). Mid-water rope nursery - testing desing and perfomance of a novel reef restauration instrument. Ecological Engineering, 36, 560-569. DOI: 10.1016/j.ecoleng.2009.12.003

Martínez, G., Alvarado, J., \& Senior, W. (2001). Estudio físico químico de las aguas superficiales de la cuenca baja y pluma del Río Manzanares. Interciencia, 26(8), 10.

MIDEPLAN. (2018). Ministerio de Planificación Naciona y Política Económica. Recuperado de http://www.mideplan.go.cr/component/content/article?id=1738

Nedimyer, K., Gaines, G., \& Roach, S. (2011). Coral Tree Nursery: An innovative approach to growing in an ocean-based field nursery. Aquaculture, Aquarium, Conservation \& Legislation International Journal of the Bioflux Society, $4(4), 442-446$.

Rinkevich, B. (2000). Steps towards the evaluation of coral reef restoration by using small branch fragments. Marine Biology, 136(5), 807-812. DOI: 10.1007/s002270000293

Rinkevich, B. (2005). Conservation of Coral Reefs through active restoration measures: recent approaches and last decade progress. Environmental Science \& Technology, 39(12), 4333-4342. DOI: 10.1021/es0482583

Rinkevich, B. (2008). Management of coral reef: we have gone wrong when neglecting active reef restoration. Marine Pollution Bulletin, 56(11), 1821-1824. DOI: 10.1016/j.marpolbul.2008.08.014

Rinkevich, B. (S.A.). The Coral Guardening Concept and the Use of Underwater Nurseries: Lesson learned from Silvic and Silviculture. In W. Precht (Ed.), Coral Reef Restoration Handbook (pp. 291-299). Israel: Taylor \& Francis. DOI: 10.1201/9781420003796.ch16

Schaffelke, B., Uthicke, S., \& Klumpp, D. (2003). Water quality, sediment and parameters at four nearshore reef flats in the Herbert river region, Central GBR. GBRMPA Research Publication, 82, 64. 
Seddon, P., Griffiths, C., Soorae, P., \& Armstrong, D. (2014). Reversing defaunatoin: Restoring species in a changin world. Science, 345(6195), 406-412. DOI: 10.1126/science.1251818

Shafir, S., Van Rijn, J., \& Baruch, R. (2006). Steps in the construction of underwater coral nursery, an essential component in reef restoration acts. Marine Biology, 149, 679-687. DOI: 10.1007/s00227-005-0236-6

Torres, M., \& Calva, L. (2012). Nutrientes en arrecifes de coral. Un caso de estudio. ContactoS, 85, 42-50.

Voss, J., \& Richardson, L. (2006). Nutrient enrichment enhances black band disease progression in coral. Coral Reefs, 25, 569-576. DOI: 10.1007/s00338-006-0131-8

Young, T. (2000). Restoration ecology and conservation biology. Biological Conservation, 92, 73-83. DOI: 10.1016/S00063207(99)00057-9 